\title{
High-Grade Glioma: Refined Diagnostics Steering Stratified Therapy
}

\author{
Martin Uhl Peter Hau \\ Department of Neurology and Wilhelm Sander NeuroOncology Unit, University Hospital \\ Regensburg, Regensburg, Germany
}

\section{Key Words}

Glioblastoma · Histology · MRI

\begin{abstract}
An adequately sampled tumor histology that is evaluated by an experienced neuropathologist is the current international standard for the diagnosis of high-grade gliomas. We present the case of a 30-year-old female for whom clinical and radiological information dramatically changed the histological diagnosis. We suggest a new algorithm including these parameters to refine the accuracy of histological diagnosis both for standard treatment and centrally reviewed clinical trials.

(C) 2013 S. Karger AG, Basel
\end{abstract}

\section{Introduction}

An adequately sampled tumor histology that is evaluated by an experienced neuropathologist is the current international standard for the diagnosis of high-grade gliomas and sets the stage for adjuvant treatment in the field of neurooncology [1]. However, standard histology, including H\&E staining, and standard immunohistochemistry markers, such as GFAP, neglect the recent development of molecular markers as well as clinical and imaging information [2].

\section{Case Report}

A 30-year-old female presented at our clinic with moderate aphasia of a few weeks' duration 14 days after she had been diagnosed with anaplastic astrocytoma based on an

Peter Hau, MD

Department of Neurology and Wilhelm Sander NeuroOncology Unit

University Hospital Regensburg, Franz Josef Strauss-Allee 11

DE-93053 Regensburg (Germany)

E-Mail peter.hau@medbo.de 
Uhl et al.: High-Grade Glioma: Refined Diagnostics Steering Stratified Therapy

open biopsy of her left temporal lobe at an external hospital. She had a 2-year history of complex focal epileptic seizures prior to diagnosis. Due to a substantial mass effect of the remaining tumor, an interdisciplinary consensus was followed, and part of the remaining tumor was resected with a substantial relief of the mass effect (fig. 1a) and almost complete recovery of aphasia, with a Karnofsky performance score of $90 \%$. The diagnosis of anaplastic astrocytoma was confirmed by the local neuropathologist, taking into consideration that some of the reactive and regressive changes (fig. $1 \mathrm{~b}-\mathrm{f}$ ) were attributable to the preceding biopsy. The presence of a mutation of isocitrate dehydrogenase (IDH1), a marker of grade II and III gliomas and secondary glioblastomas, substantiated the diagnosis [3]. After central review of the histology by 2 reference neuropathologists, randomization into an international clinical trial for anaplastic gliomas was rejected due to the fact that necrosis in the histological samples obtained during the second surgery qualified, in the view of the reference neuropathologists, as glioblastoma. At that point, considering the size of the residual tumor mass and in view of the clinical history and an MRI suggestive of a rather aggressive high-grade glioma, we decided to start the patient on radiochemotherapy with temozolomide according to the Stupp protocol [4].

\section{Conclusions}

We believe that this case illustrates some of the limitations of the current process of how diagnoses for patients with high-grade gliomas are made. First, a tumor biopsy, open or stereotactic, selects a small portion of the tumor which may not necessarily represent the total tumor mass. Second, molecular information, such as IDH1 mutation, can add substantial information to the kind and dignity of the tumor. Third, information from MRI and the dynamics of the clinical course may substantially differ from the histological information from the tumor biopsies and may enhance a comprehensive evaluation of the individual course. And fourth, local and particularly central review neuropathologists are often not adequately supported with clinical and imaging information to make a conclusive diagnosis.

Since a change in the diagnosis implicates a substantial change in the treatment for the affected patient, we therefore mandate gross total resections, whenever applicable, to obtain a maximal amount of tumor material, along with the integration of imaging and clinical information in the material submitted to local and central neuropathology [5]. We think that the minimal time delay incurred before treatment by establishing such an algorithm would be negligible; the algorithm would favor diagnostic accuracy and advance both standard treatment and the homogeneity of study populations within centrally reviewed clinical trials.

\section{Acknowledgement}

The histological images are a courtesy of Prof. M.J. Riemenschneider, Department of Neuropathology, University of Regensburg, Germany. The MRI scans are courtesy of Prof. G. Schuierer, Center for Neuroradiology, University Hospital and County Hospital, Regensburg, Germany.

\section{References}

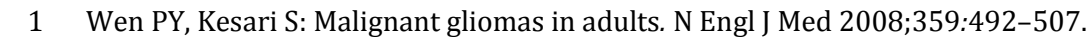


2 Weller M, Stupp R, Hegi ME, et al: Personalized care in neuro-oncology coming of age: why we need MGMT and 1p/19q testing for malignant glioma patients in clinical practice. Neuro Oncol 2012;14(suppl 4):iv100iv108.

3 Yan H, Parsons DW, Jin G, et al: IDH1 and IDH2 mutations in gliomas. N Engl J Med 2009;19:765-773.

4 Stupp R, Mason WP, van den Bent MJ, et al: Radiotherapy plus concomitant and adjuvant temozolomide for glioblastoma. N Engl J Med 2005;352:987-996.

5 Wesseling P, Kros JM, Jeuken JWM: The pathological diagnosis of diffuse gliomas: towards a smart synthesis of microscopic and molecular information in a multidisciplinary context. Diagn Histopathol 2011;17:486494. 


\section{Case Reports in Oncology}

\begin{tabular}{l|l}
\hline Case Rep Oncol 2013;6:387-390 \\
\hline DOI: $10.1159 / 000353887$ & $\begin{array}{l}\text { C 2013 S. Karger AG, Basel } \\
\text { www.karger.com/cro }\end{array}$ \\
\hline
\end{tabular}

Uhl et al.: High-Grade Glioma: Refined Diagnostics Steering Stratified Therapy
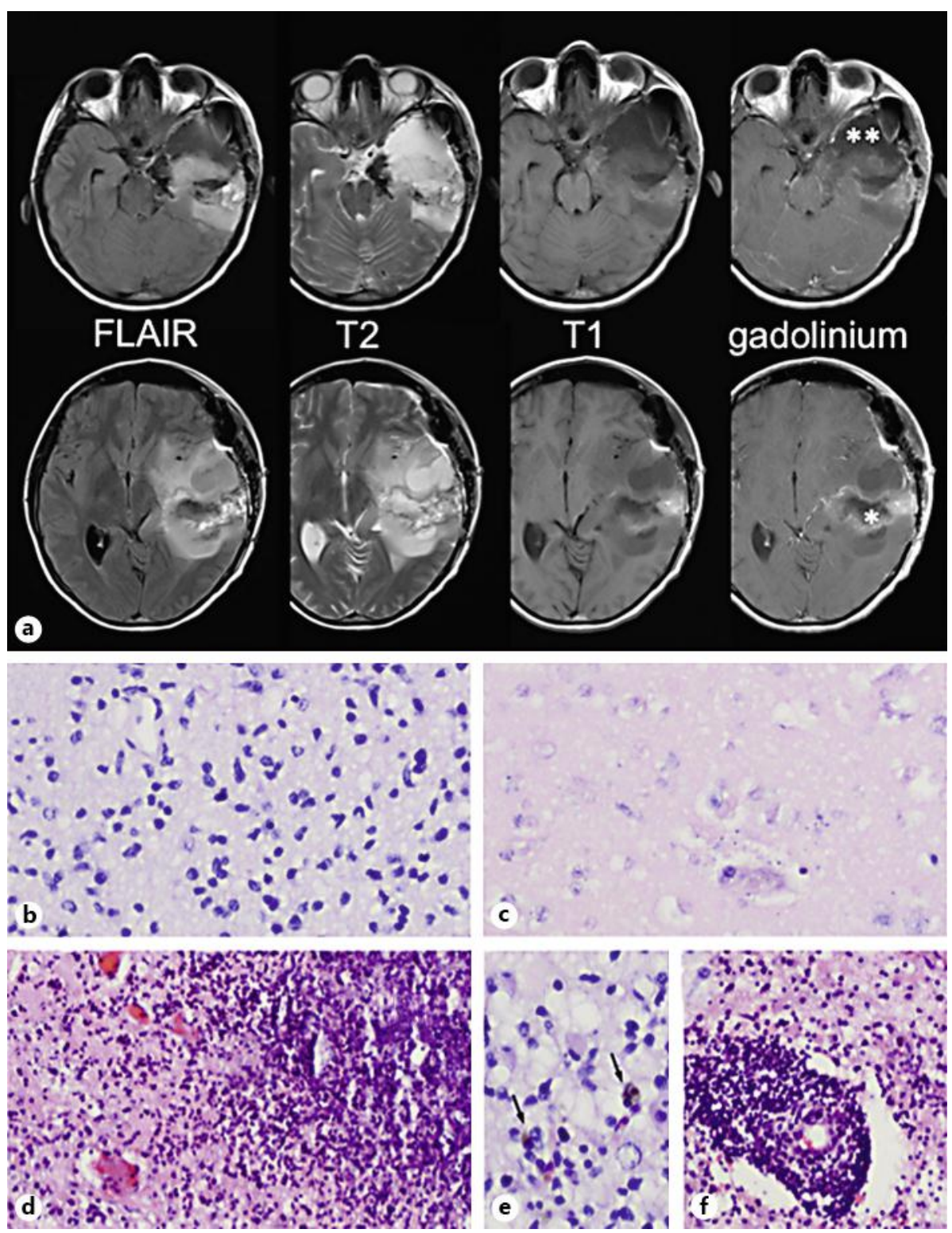

Fig. 1. a Postoperative MRI scan performed within $24 \mathrm{~h}$ after the second resection. FLAIR, T2 and T1 sequences with and without contrast-enhancing gadolinium. The single asterisk $(*)$ marks the initial external hospital biopsy site, whereas the double asterisk $\left(^{* *}\right)$ marks the area of the gross resection at our institution. There is pronounced gadolinium enhancement in the dorsal parts of the tumor, including necrotic areas and signs of hypervascularization. $\mathbf{b}-\mathbf{f}$ Histology of the gross resection. $\mathbf{b}$ Most parts of the tumor appear as anaplastic astrocytoma, lacking necrosis or vascular proliferation. In some parts, histological changes were detected (c-f) that could most likely be attributed to the prior stereotactic biopsy: focal areas of surrounding necrotic non-neoplastic brain tissue with low cellularity (c); necrotic tumor areas intermixed with granulocytic infiltrates (d); hemosiderin deposits as a sign of a previous hemorrhage (arrows, e), and reactive inflammation surrounding blood vessels (f). 